\title{
Spinal cord injury hospitalisation in a rehabilitation hospital in Japan
}

\author{
Y Hasegawa MSW,${ }^{1} \mathrm{M}$ Ohashi MD,${ }^{1 *} \mathrm{~N}$ Ando MD,${ }^{1} \mathrm{~T}$. Hayashi MD,${ }^{1} \mathrm{~T}$ Ishidoh MD,${ }^{1}$ \\ K Kumagai MD, ${ }^{2}$ A Shimazu ${ }^{2}$ \\ ${ }^{1}$ Kanagawa Rehabilitation Hospital, ${ }^{2}$ Research Institute of The Kanagawa Rehabilitation \\ Center, 516 Nanasawa, Atsugi-City, Kanagawa-Prefecture, Japan.
}

The Kanagawa Rehabilitation Hospital was established by Kanagawa Prefecture 20 years ago. The hospital has a 40 bed SCI unit and has been providing comprehensive medical and rehabilitation services for SCI patients of this prefecture.

A letter of enquiry to 404 major surgical units within this prefecture revealed that $630 \mathrm{SCI}$ patients had been under care in this prefecture during a period of 3 years. Of these, the Frankel grade $\mathrm{A}, \mathrm{B}$ and $\mathrm{C}$ patients, were assumed to be rehabilitation candidates, totalling about 130 in this prefecture per year. The inpatient medical record revealed that we treated 770 SCI patients from 1986 to 1991. From this data, we estimated that the hospital accepted an average of 54.5 SCI patients per year for rehabilitation purposes. Other inpatients were rehospitalised for various complications. According to the study of 125 patients who were hospitalised for comprehensive rehabilitation, they waited an average of 139 days and stayed in the hospital for 288 days. The increasing number of chronic patients who required treatment of complications appeared to be delaying the early admission of rehabilitation patients. The medical treatment cost for those with spinal cord injuries was less than that for the average patient with other types of medical conditions.

Keywords: spinal cord injury; incidence; rehabilitation; complications; costs of hospitalisation.

\section{Introduction}

Early initiation of the medical and rehabilitation treatment that are specially organised for patients with traumatic spinal cord injuries (SCI) is the key for the effective management of such patients. Several Western countries have arranged regional spinal cord injury care systems for this purpose. In Japan such a well organised system is not available, therefore SCI patients are usually treated in the emergency care units of general hospitals. If the patients have complicated medical problems, they are referred to other hospitals where better equipment and staff can provide more intensive medical treatment. After the acute medical status has been stabilised, patients desire the opportunity of having rehabilitation.

${ }^{*}$ Correspondence.
The medically well equipped hospitals do not always have a comprehensive rehabilitation service, and well recognised rehabilitation hospitals are not always able to admit emergency patients. Keeping this setting in mind, this paper presents the results of an analysis of SCI hospitalisation in a rehabilitation hospital.

\section{Method}

Kanagawa Prefecture is adjacent to the capital city, Tokyo, and the population was $8,149,282$ in 1992. The Kanagawa Rehabilitation Center was established by the prefecture in 1973 and owns two hospitals, seven welfare facilities, a research institute and a nursing school. This rehabilitation center is very well equipped in terms of rehabilitation staff, with physical therapists, occupational 
therapists, social workers, vocational counsellors, rehabilitation engineers and rehabilitation gymnasts. The Kanagawa Rehabilitation Hospital is one of the two hospitals in the center, and has a 40 bed SCI unit, which is run by orthopaedic surgeons, urologists, and rehabilitation medicine physicians. The hospital budget is partially aided by the prefecture, so the hospital admits mostly residents of Kanagawa Prefecture.

To establish the incidence of SCIs in this prefecture, we mailed a questionnaire to 404 major surgical units within the prefecture during 3 consecutive years from 1990 to 1992. A response was obtained from $58.1 \%$ of the facilities, which included major trauma centers and university hospitals.

We next looked into the medical records of 770 SCI patients who were admitted to our spinal cord injury unit during 5 consecutive years from 1986 to 1991. These 770 patients had treatment for SCI related complications and they also had rehabilitation. From this data, the status of the bed utilisation of this unit was assessed.

Lastly, to know the status of SCI treatment in this hospital, we selected 125 patients. They were hospitalised for comprehensive rehabilitation treatment during 33 consecutive months from June 1989 to February 1992. All patients had physical impairments and disabilities from traumatic spinal cord injuries. The patients were grouped into four units according to the time from injury to admission (Table I). Their hospitalisation days and Barthel Index scores on admission and discharge were studied. Furthermore, the hospital charge of a single day for each group and the average hospital charge of a nearby prefectural general hospital were compared.

\section{Results}

The results of the survey revealed that 630 SCI patients had been cared for in this prefecture during a 3 year period. There were 533 male patients with an average age of 40.7, and 97 female patients with an average age of 46.2 . The male to female ratio was 5.5:1. The causes of the injuries were motor vehicle accidents $44.8 \%$, falls from heights $26.8 \%$, falls on the ground $9.0 \%$, sports accidents $6.0 \%$, being hit by objects $5.4 \%$, falls from attempted suicide $4.3 \%$, and others $3.7 \%$. According to Frankel's neurological classification, 630 patients were divided into five groups. From the rehabilitation standpoint, $\mathrm{A}, \mathrm{B}$ and $\mathrm{C}$ group patients were candidates for rehabilitation treatment because they had significant physical impairment and disabilities. There were 212 group A, 62 group B and 125 group $\mathrm{C}$ patients. The total of $\mathrm{A}, \mathrm{B}$ and $\mathrm{C}$ patients during the 3 years was 399 . This indicates that there was an average $133 \mathrm{SCI}$ rehabilitation candidates in this prefecture per year.

Of the 770 patients who were hospitalised in the spinal cord injury units from 1986 to 1991, 272 were admitted for rehabilitation treatment. Therefore, our hospital accepted an average of $54.5 \mathrm{SCI}$ patients per year for the purpose of rehabilitation. Others were chronic patients who were rehospitalised for the treatment of complications. One hundred and seventy nine patients required pressure ulcer treatment, and 147 required various urological treatments. Also, 172 patients were rehospitalised with a variety of medical complications. Figure 1 shows the composition of the number of patients and the days of bed occupancy in our SCI unit.

The 125 patients who were hospitalised for rehabilitation treatment had to wait for admission to the hospital for an average of 139 days and stayed in the hospital for an average of 149 days. They were grouped into four units according to the number of waiting days (Table I and Figure 2). The average hospitalisation of group 4 was 30 days shorter than that of group 1. Yet they had to be hospitalised for an average of 365 days, if the days are counted from the onset of the SCI. Paraplegics stayed in the hospital for an average of 128 days, and their total hospitalisation days were 279. Tetraplegics stayed in this hospital for an average of 179 days and their total hospitalisation days were 300 . Figure 3 shows the ADL achievement of groups 1 and 4 (complete paraplegics) on admission and discharge. Group 1 patients were dependent in selfcare 
Table I Rehabilitation patients grouped by length of waiting interval

\begin{tabular}{lccccc}
\hline & \multicolumn{2}{c}{ Paraplegic } & \multicolumn{2}{c}{ Tetraplegic } & \\
\cline { 2 - 4 } & Complete & Incomplete & Complete & Incomplete & Total \\
\hline Group 1 & 9 & 2 & 4 & 4 & 19 \\
Group 2 & 7 & 2 & 8 & 2 & 19 \\
Group 3 & 10 & 1 & 6 & 3 & 20 \\
Group 4 & 32 & 9 & 15 & 11 & 67 \\
Total & 58 & 14 & 33 & 20 & 125 \\
\hline
\end{tabular}

Waiting interval

Group 1: $<7$ days.

Group 2: $>7$ days, $<6$ weeks.

Group 3: $>6$ weeks, $<12$ weeks.

Group 4: $>13$ weeks.

\section{Numbers of patients}

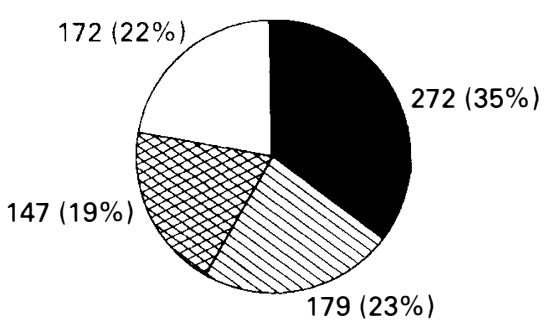

Days of bed occupancy

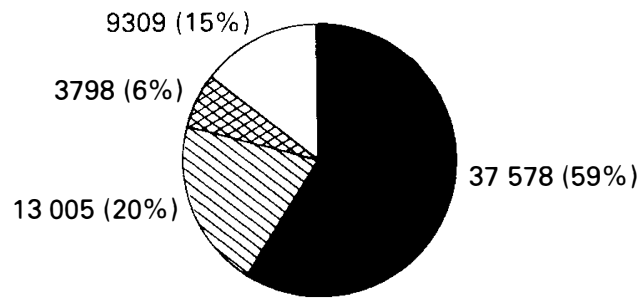

Rehabilitation

$\triangle$ Pressure ulcers

$\otimes$ Urological complications

Others

Figure 1 Composition of 770 patients in the SCI unit from 1986 to 1992.

activities on admission. They became independent in these activities at discharge. Group 4 patients were independent in most of the selfcare activities except bathing, toileting, and transfer activities on admission. They achieved independence in a shorter number of days than group 1 . Groups 1, 2 and 3 (complete tetraplegics) required 7.5 months to achieve functional goals and preparations to live at home. Group 4 (complete tetraplegics) required 6 months to reach the same goals. Figure 4 shows the average hospital charge per day for four groups. Group 1 paid the largest medical treatment charges compared to other groups. This is because, as acute patients, group 1 patients required more

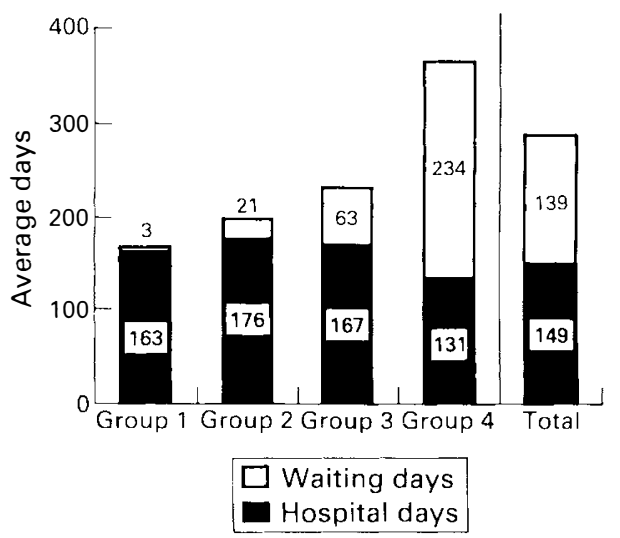

Figure 2 Average waiting days and hospital days of 125 patients for rehabilitation treatment. 


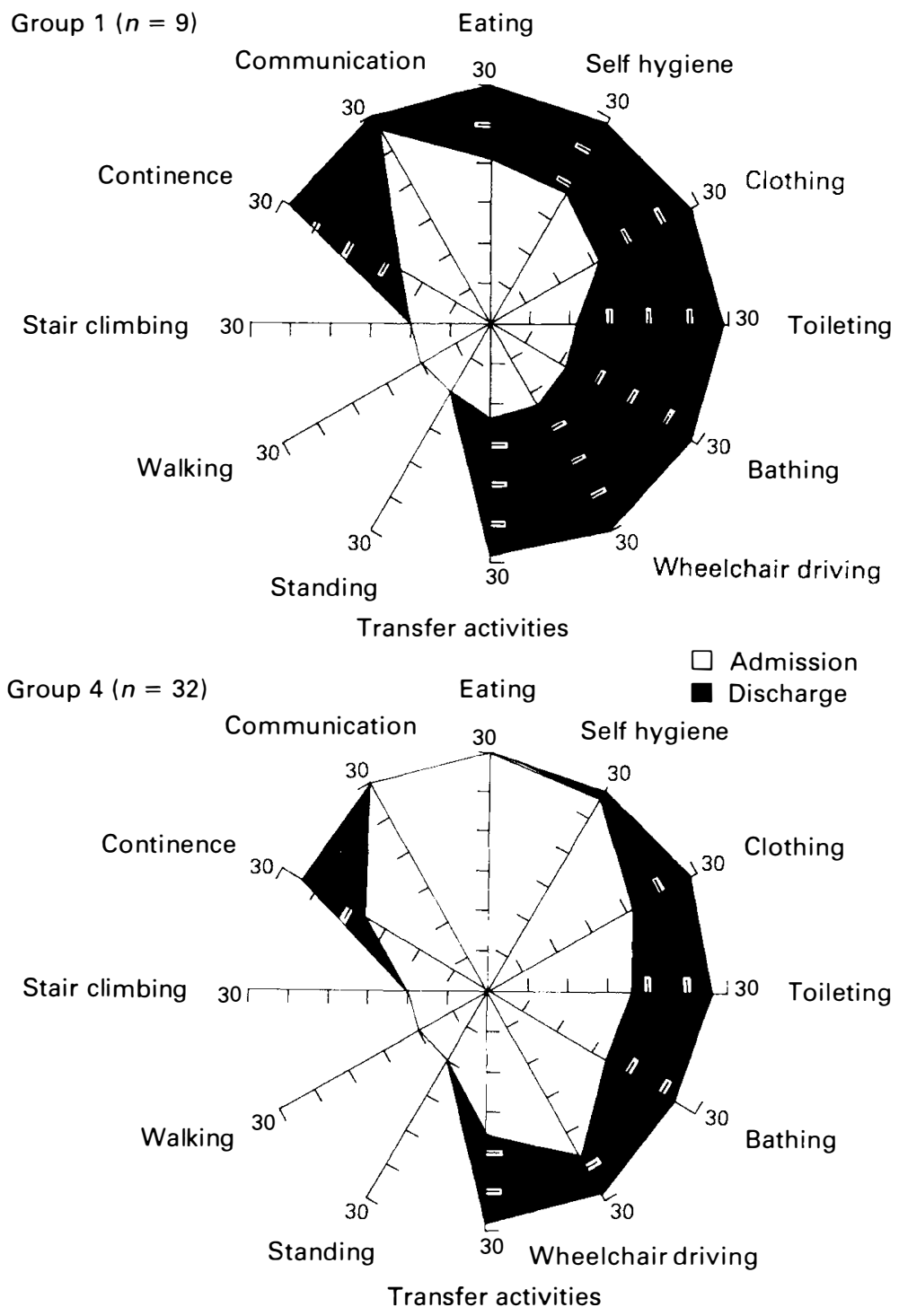

Figure 3 ADL achievement of complete paraplegics. (Comparison of group 1 and group 4). Barthel subscores were modified to show in radar charts. Complete independence $=30$. Partial assistance $=20$. Complete dependence $=10$.

medical examinations and treatments than did patients in the other groups. Figure 5 shows our hospital charges for spinal cord injuries and other conditions and also presents the hospital charges for average patients in a nearby general hospital. The medical treatment fee for spinal cord injuries is less than that of the average patients who were treated for other medical conditions in a general hospital. At the time of the survey, the currency exchange rate of the US dollar was 129.3 yen.

\section{Discussion}

The incidence study revealed that there were about 130 severely disabled SCI patients in this prefecture per year. All 


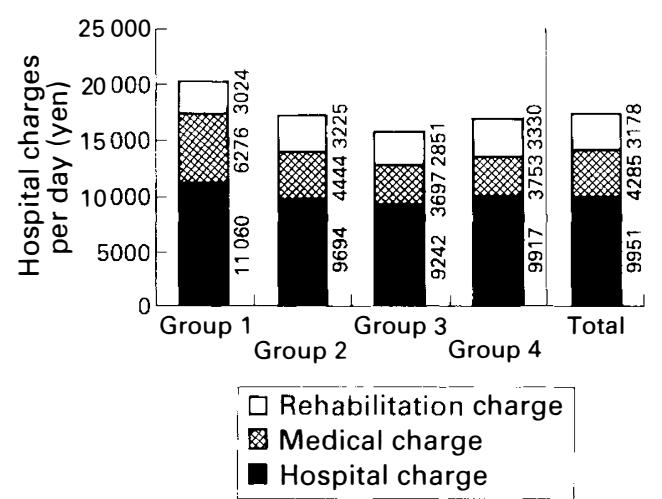

Figure 4 Average hospital charge of SCI rehabilitation patients of different waiting intervals.

required comprehensive rehabilitation. Not all patients are referred to the Kanagawa Rehabilitation Hospital, some go to other rehabilitation facilities. From all of the referred patients, some are rejected because of age, medical complications, or poor social background. Some 40-50 patients per year are usually accepted as rehabilitation patients in our hospital. They require to wait about 4 months until a bed becomes available. Oakes et $a l^{1}$ and Devivo et $a l^{2}$ stress the importance of early admission to comprehensive rehabilitation units. Early treatment in a special SCI unit can offer better results, with a shortening of the total length of hospitalisation, avoidance of complications and a reduction of medical costs. These results are undoubtedly correct, but in our experience SCI patients tend to return to the SCI unit seeking treatment for complications. During the past 20 years, the number of SCI patients in our hospital has increased. Some develop pressure ulcers or urological complications, and others develop geriatric disorders. They are reluctant to go to other general hospitals and prefer to come to a rehabilitation hospital because in such a hospital the medical staff are quite familiar with the management of SCI patients. It appears that to shorten the number of waiting days for the admission of
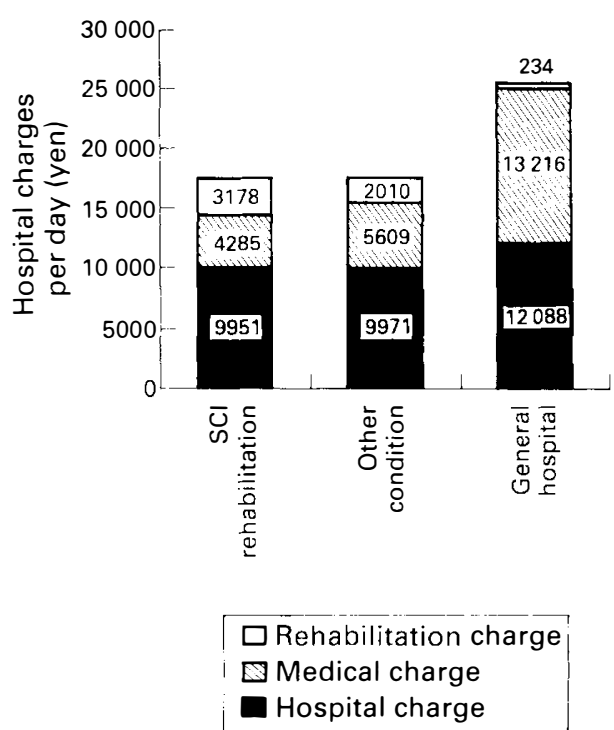

Figure 5 Average hospital charge of SCI rehabilitation patients and other conditions.

new patients to our hospital, we should either increase the number of beds or reject the chronic patients with complications. Another possibility is that the SCI prevention campaign must be enforced to reduce the number of potential SCI patients.

As for the financial cost of hospitalisation, all Japanese people are covered by the government health insurance system. From the patient's standpoint, this system minimises the economic burden for every person. However, long and ineffective hospitalisation places enormous stress on the national budget. From the rehabilitation hospital's standpoint, the government system only pays for the physical and occupational therapy, not for the rehabilitation prescription fee of rehabilitation specialists, nor for the work of social workers, vocational counsellors, prevocational training specialists and rehabilitation gymnasts. This results in lower hospital charges for SCI patients compared to patients with other medical conditions.

\section{References}

1 Oakes DD, Wilmot CB, Hall KM, Sherch JP (1990) Benefits of early admission to a comprehensive trauma center for patients with spinal cord injury. Arch Phys Med Rehabil 171: 637-643.

2 Devivo MJ, Kartus PL, Stover SL, Fine PR (1990) Benefits of early admission to an organized spinal cord injury care system. Paraplegia 28: 545-555. 